\title{
System Simulation and Implementation of SIPS in Taiwan
}

\author{
Chao-Rong Chen, Wen-Ta Tsai, and Hua-Yi Chen \\ National Taipei University of Technology, 1, Sec. 3, Zhongxiao E. Road, Taipei 10608, Taiwan \\ Correspondence should be addressed to Wen-Ta Tsai; s2319002@ntut.edu.tw
}

Received 20 February 2014; Accepted 8 April 2014; Published 22 May 2014

Academic Editor: Her-Terng Yau

Copyright (c) 2014 Chao-Rong Chen et al. This is an open access article distributed under the Creative Commons Attribution License, which permits unrestricted use, distribution, and reproduction in any medium, provided the original work is properly cited.

\begin{abstract}
This study describes the system simulation and implementation of the system integrity protection schemes (SIPS) in an independent, intensive, and island-type power system. It also elucidates a smart grid plan to provide grid security in this power grid. The proposed SIPS can prevent blackouts that could otherwise result from the transient instability of N-3 contingencies and has been fully implemented and operated. The entire SIPS installation comprises two stages. The first-stage SIPS takes generator tripping system simulation and the second-stage SIPS involves generator tripping, load rejection, and bus-tie switching countermeasures. The proposed SIPS can prevent isolated power system blackout from extreme contingencies system and provide a valuable system simulation experience for similar independent power grids.
\end{abstract}

\section{Introduction}

The electrical power infrastructures of Taiwan are decades old. The grid is aging and stressed. The construction of new transmission lines and substations has been postponed because of environmental protests, reducing reliability. These phenomena affect the reliability of Taiwan power system. Taiwan Power Company (TPC) is suggested to build system integrity protection schemes (SIPS) to improve the reliability and furthermore places SIPS into the grid security goal in its smart grid milestone. TPC has developed smart grid in 2006. Its four goals which are grid safety and reliability, electric energy efficiency, customer service, and distribution resources integration are presented in Figure 1. These goals include four, three, two, and two items, respectively. Each item contains some subitems. The SIPS is an advanced system protection subitem that is included in the A1 item. Smart grid is expected to provide self-correction, reconfiguration, and restoration and to handle the randomness of loads and market participants in real time. It is expected to have key characteristics that self-healing; empower and incorporate that consumer, and so forth [1].

There are some large blackouts worldwide caused by system-wide disturbances in the past decade [2,3]. The tight operating margins and less redundancy are the common situation for power system. These phenomena make the power system more complex to operate. Therefore, an automated scheme is needed to detect system abnormal conditions that probably cause system stress and take predetermined actions. The basic changes in planning and operation of the power system require complete system protection solution to counteract disturbance propagation. The SIPS includes special protection systems (SPS) and remedial action schemes (RAS), as well as other system integrity schemes, such as under-frequency (UF), under-voltage (UV), and outof-step (OOS) [4].

SIPS are one of the essential countermeasures due to their relative low cost and shorter installation. The purposes of SIPS are preserving stabilities and preventing overload as well as system oscillations [5]. SIPS application examples are overload mitigation, system separation, undervoltage load shedding, and so on [6]. SIPS are also used to manage system disturbances and prevent blackouts [7, 8]. TPC is implementing the smart grid using a real-time SIPS installation with event based characteristics.

SIPS are commonly referred to as remedial action schemes (RAS) or special protection systems (SPS) that were being widely employed. For example, SPS features in optimized defense plan against bus-bar faults at some substations [9], maximizing scheduling generation capacity 


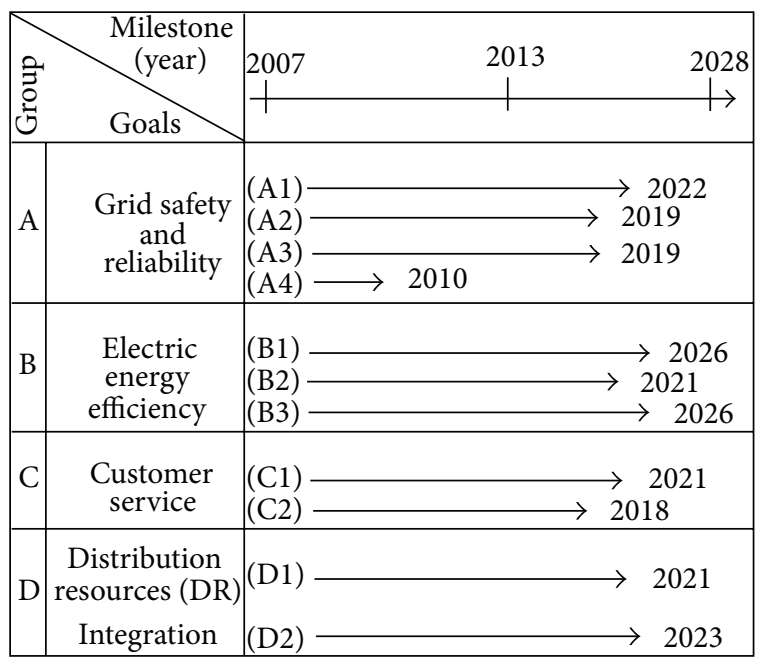

FIGURE 1: Smart grid milestone and group goals in TPC. A1: stability and reliability enhancement,A2: intelligent operation, A3: application on advanced power electronics devices,A4: preventive maintenance technology, B1: generator efficiency, B2: grid efficiency, B3: operation efficiency, C1: smart meter and billing, C2: valueadded service, D1: DR prediction and operationD2: DR integration.

[10], preventing power congestion [11], maintaining security at high import power transfer [12], and preventing power failure $[13,14]$. One of the most famous SIPS applications is the Hydro-Quebec defense plan which is against extreme contingencies [15]. For this paper, the term SIPS will be used instead of SPS or RAS. For the last decade, large grids blackout occurred that led to unexpected consequence. Clearly, power system outage seems to be inevitable, but the probability of blackout must be reduced. This work studies efforts to avert blackouts in an isolated and intensive power system, based on the lessons learned from previous blackouts [16]. SPS are used to involve local operational constraints, but this work proposes the implementation of system-wide SIPS to prevent transient instability of N-3 contingencies in Taiwan power system.

To improve reliability and prevent blackouts, TPC started to plan SIPS after the previous outage in 1999. The systemwide SIPS has two stages, as presented in Figure 2. The first-stage SIPS is designed to prevent some specific N-3 contingencies and controlled by operators in central dispatching control center (CDCC) and power plant control room. The second-stage SIPS is designed to prevent all N-3 contingencies with automation; it performs generator rejection, load shedding, and bus-tie switching. Both of these two stages solve the problem of transient instability caused by N-3 contingencies. Transient instability was one of the main causes for the blackout in 1999. The defensive strategy is to install system-wide SIPS with event-based action tables. Power system planners apply SIPS to protect power system against the following issues [2]:

(1) congestion;

(2) small-disturbance angle instability;

(3) transient instability;

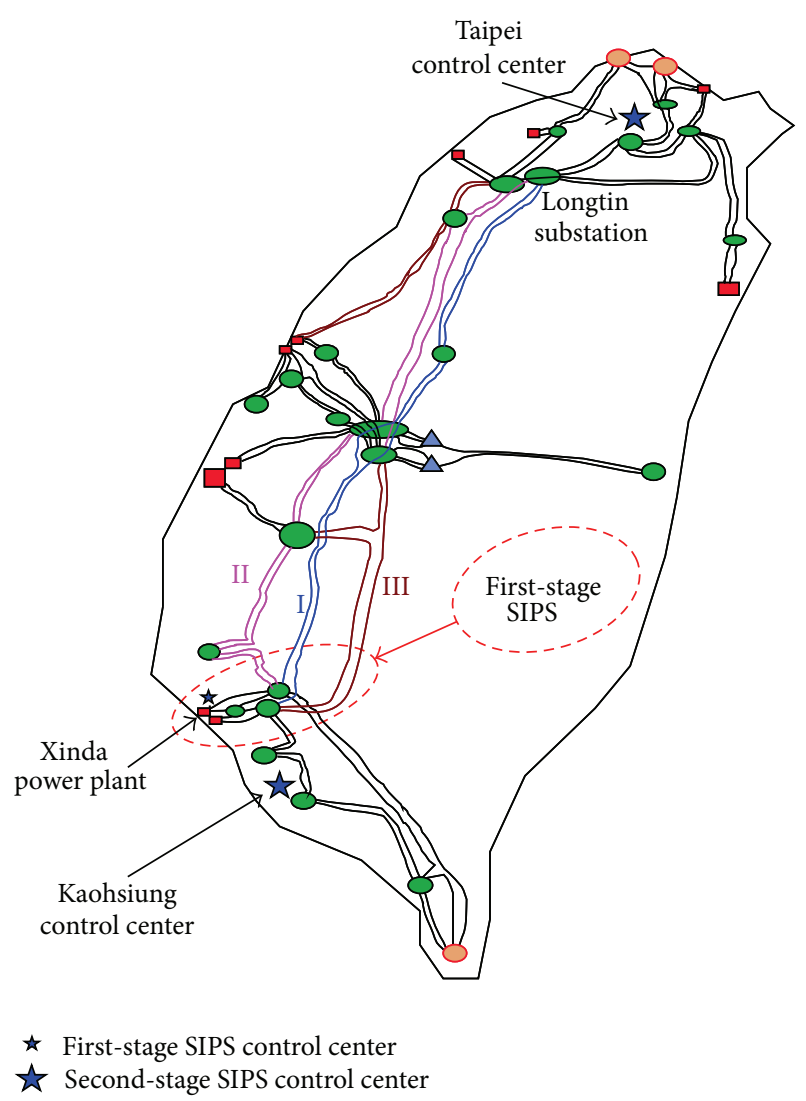

FIGURE 2: The configuration and location for the two-stage SIPS in Taiwan.

(4) frequency instability;

(5) voltage instability;

(6) thermal overloading.

SIPS in this paper meets issue (3). The architecture is system-wide with redundancy design consideration, taking event based algorithm for decision-making process. The simulation results are called look-up tables, which provide the SIPS actions. SIPS action times must be completed within 250 milliseconds after fault clearance.

\section{Taiwan's Power System}

The power system in Taiwan is of the island-type, intensive, and isolated without any connection to another grid. It is managed by Taiwan Power Company (TPC). TPC is a vertically integrated and government-owned electrical power company. Its installation capacity is $41.06 \mathrm{GW}$ and its peak load in 2013 was around $34.3 \mathrm{GW}$. The transmission system is supplied by 21 major power plants that have more than 100 generator units. They are including nuclear, coal, oil, combined-cycle, hydro-, and cogeneration plants, representing $20 \%, 43 \%, 5 \%, 23 \%, 4 \%$, and $5 \%$, respectively, of generation capacity. The extra high voltage level is $345 \mathrm{kV}$ with a total circuit length of $3,686 \mathrm{~km}$, and the high voltage transmission is $161 \mathrm{kV}$ with a total circuit length of $6,203 \mathrm{~km}$. The 
TABLE 1: Brief introduction for Taiwan power system.

\begin{tabular}{lc}
\hline \multicolumn{2}{c}{ Generation capacity percentage by fuel } \\
\hline Nuclear & $20 \%$ \\
Coal & $43 \%$ \\
Oil & $5 \%$ \\
Combined-cycle & $23 \%$ \\
Hydro & $4 \%$ \\
Renewable & $5 \%$ \\
\hline \multicolumn{2}{c}{ Transmission line circuit length $(\mathrm{km})$ by voltage level } \\
\hline $345 \mathrm{kV}$ & 3,686 \\
$161 \mathrm{kV}$ & 6,203 \\
$69 \mathrm{kV}$ & 6,168 \\
$11 / 22 \mathrm{kV}$ & 316,680 \\
Peak load [date] & $34.3 \mathrm{GW}[$ Aug. 9,2013$]$ \\
Installation capacity & $41.06 \mathrm{GW}$ \\
\hline
\end{tabular}

subtransmission voltage level is $69 \mathrm{kV}$, and the corresponding total circuit length is $6,168 \mathrm{~km}$. The voltage levels of distribution network are $11 \mathrm{kV}$ and $22 \mathrm{kV}$, and the corresponding total circuit length is $316,680 \mathrm{~km}$ [17]. The brief introduction for Taiwan power system is shown in Table 1. The northern area is the load center and the central and southern areas are generation centers. Taiwanese grid is characterized by large power transfer from south to north in periods of peak load, which promotes transient instability problem following large disturbances, against which the system-wide SIPS measures, described herein, are implemented.

\section{The First-Stage SIPS}

The first-stage SIPS was motivated by the power outage in 1999, which was caused by the crashing of an EHV tower and led to transient instability [16]. That event led to approximately $83 \%$ of consumers without electricity. The power outage had two main causes. One is the excess south-to-north (STN) power transfer, and the other is the delay associated with the third EHV transmission trunk shown as III in Figure 2. The STN power transfer via two EHV transmission trunks in the power outage of 1999 was large. Thereafter, TPC decided to upgrade its security and reliability by adopting the suggestion in the final report of the blackout event in 1999. Figure 3 presents the generator tripping scenario for the first-stage SIPS. The generator tripping scheme will be initiated for any three lines of line A to line $\mathrm{F}$ fault or outage; it depends on the STN power flow. For the first SIPS experience and conservative consideration, we suggest the two levels operators which include the central dispatch and plant control centers to monitor and operate.

3.1. Transient Stability. We can obtain N-3 contingencies with the combination of line $\mathrm{A}$ to $\mathrm{F}$ as in Figure 3 . The N-3 contingency means normal grid lost (-) 3 elements which are 3 transmission lines in this study. The N-3 includes the combination of the N-2-1 and N-1-2. From the combination

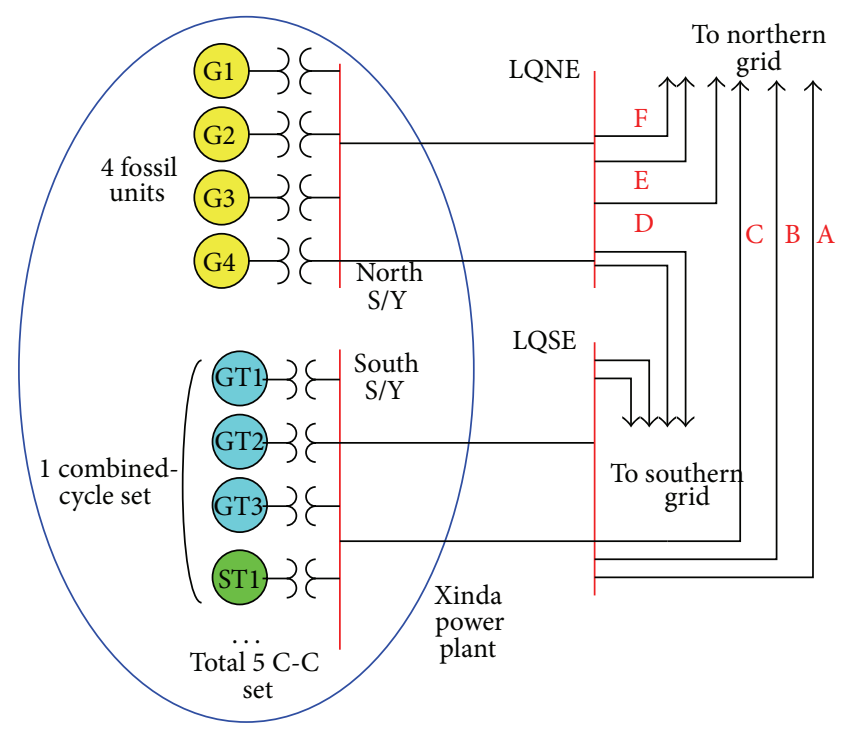

FIGURE 3: Generators tripping scenario for the first-stage SIPS.

theory, there are 60 combinations for N-2-1 and N-1-2 with each STN transfer as follows:

$$
\begin{aligned}
& \mathrm{N}-2-1, \mathrm{~N}-\mathrm{X}-\mathrm{Y}, \mathrm{X}=\mathrm{C}(6,2)=15, \mathrm{Y}=\mathrm{C}(4,1)=4, \\
& \mathrm{~N}-1-2, \mathrm{~N}-\mathrm{X}^{\prime}-\mathrm{Y}^{\prime}, \mathrm{X}^{\prime}=\mathrm{C}(6,1)=6, \mathrm{Y}^{\prime}=\mathrm{C}(5,2)=10 .
\end{aligned}
$$

Then we simulate N-3 contingencies with three-phase fault as well as different STN transfers to examine transient instability. Table 2 illustrates the simulation results. The division of the STN power transfer on Table 2 is $100 \mathrm{MW}$, that is, easier index for operators. There are two kinds of line capacity for line A to line F in Figure 3. The capacity of line A and line D is $1200 \mathrm{MW}$ and the others are $2200 \mathrm{MW}$. For this reason, $3700 \mathrm{MW}$ STN transfer flow is a base for simulation. It is not necessary to act for first-stage SIPS as long as the STN transfer is below $3700 \mathrm{MW}$. The action conditions of this SIPS are the STN transfer being greater than $3701 \mathrm{MW}$ with $\mathrm{N}-1-2 / \mathrm{N}-2-1$. The logic for tripping generators is shown as in Figure 4. The SIPS is taking the preventive measures. When the STN flow is greater than $3701 \mathrm{MW}$ or N-1-2/N2-1 happening, the SIPS is in arming condition. The CDCC operator immediately commands plant operator to turn on generator rejection switches shown as in Figure 7. This can respond to the probably following contingency.

3.2. Small-Signal Stability. In order to ensure other stability problems, the small-signal stability and voltage stability must be checked. Generally, installing the power system stabilizer is one of the effective and economic ways to improve system damping characteristics. The simulation software is EPRI PEALS which is frequency domain algorithm. Table 3 shows the simulation results including the comparison between the normal and $\mathrm{N}-3$ contingency conditions.

As the STN transfer increases, however, the damping ratio decreases in both normal and N-3 contingency conditions. This indicates that the greater the interarea power transfer, the weaker the system characteristics. The damping ratio 
TABLE 2: Action table including judgment and action.

\begin{tabular}{lc}
\hline $\begin{array}{l}\text { Judgment } \\
\text { (STN transfer MW) }\end{array}$ & $\begin{array}{c}\text { Action } \\
\text { (GT rejecting number) }\end{array}$ \\
\hline$<3700$ & 0 \\
$3701 \sim 3800$ & 2 \\
$3801 \sim 3900$ & 5 \\
$3901 \sim 4000$ & 7 \\
$4001 \sim 4100$ & 10 \\
$4101 \sim 4200$ & 11 \\
$4201 \sim 4300$ & 13 \\
$>4301$ & 15 \\
\hline
\end{tabular}

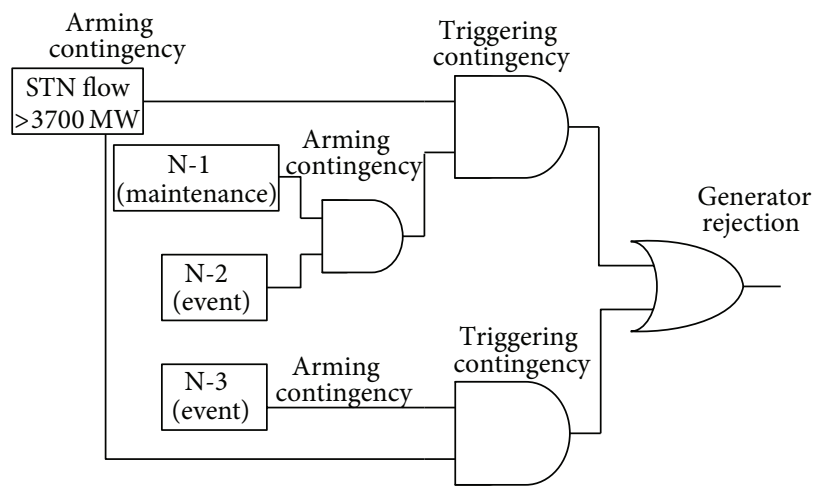

FIGURE 4: The logic for tripping generators of the first-stage SIPS.

range is from $4.28 \%$ to $5.01 \%$ and $2.92 \%$ to $3.75 \%$ for normal and N-3 contingency situation, respectively. The damping ratio values are valid according to the planning criteria for the Taiwan Power Company transmission system [18]. The oscillation frequencies are approximately $1.2 \mathrm{~Hz}$; that means the oscillation belongs to interarea mode. The STN power transfer and damping ratio reveal an inverse linear relationship. Therefore, the small-signal stability is not the main constraint for this isolated power system.

3.3. Voltage Stability. Heavy power transfer with long distance transmission and fault often results in voltage problem. Therefore, the relationship between the increasing STN power flow and voltage stability is examined. The EPRI VSTAB software is used for simulations. The simulation procedure is to decrease the northern area generation and increase the same quantity generation in the south by $100 \mathrm{MW}$ for each step to transfer more STN power flow. The bus voltage of LUNCHI-N substation is the lowest after screening all $345 \mathrm{kV}$ buses. Figure 5 shows the P$\mathrm{V}$ curve of LUNCHI-N substation for both the normal and N-3 contingency conditions. To compare the results of transient stability simulation, the voltage stability has a higher operating margin. For example, Table 2 shows that the increment for the STN power flow in transient stability is around $600 \mathrm{MW}$ (from 3700 to $4300 \mathrm{MW}$ ). However, the incremental increase is about $800 \mathrm{MW}$ (e.g., from $10250 \mathrm{MW}$

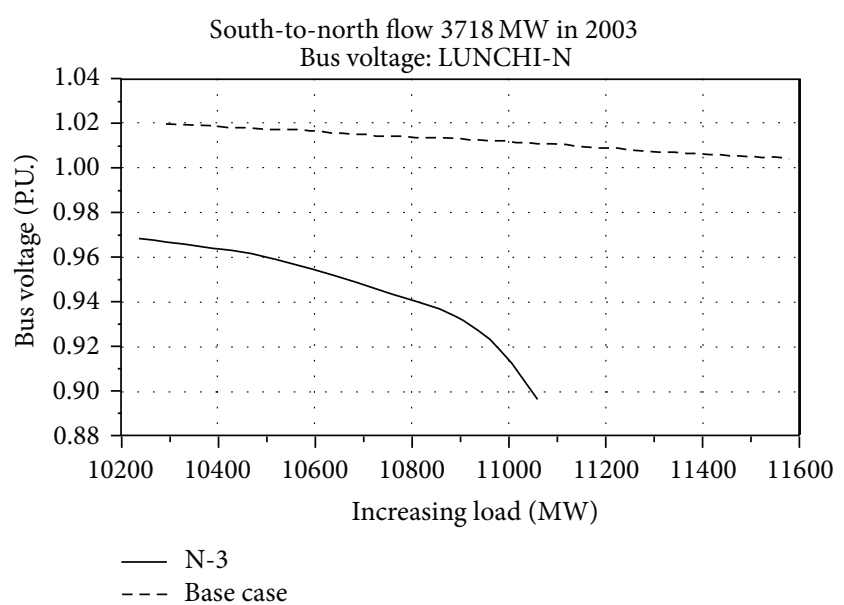

FIGURE 5: The P-V curve for the real power transferring ability and voltage margin at LUNCHI-N substation.

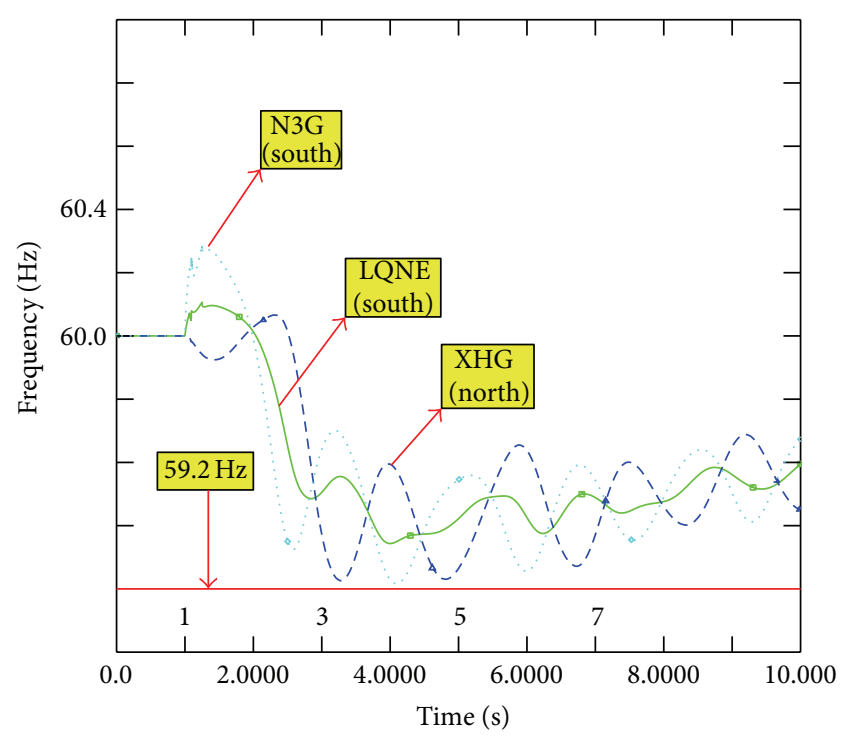

FIGURE 6: Frequency swing as tripping thirteen generators.

to $11050 \mathrm{MW}$ ) as in Figure 5. Consequently, voltage stability has a larger operating margin than transient stability.

3.4. Frequency Response. Electric power system should install under-frequency load shedding (UFLS) scheme to be the last defensive line against extreme contingencies. It is important to examine the system frequency response while rejecting the maximum thirteen generators whether reach the UFLS setting. The largest stable STN power transfer $4300 \mathrm{MW}$ is used to simulate frequency response; that is, total rejection generation is around $1641 \mathrm{MW}$. From Figure 6, we simulated a 3-phase fault happening at 1 second at LUNCHI-N substation and the fault was cleared after 4.5 cycles. There are three frequencies swinging which represent the southern, central, and northern buses. The simulation result shows these frequencies swing down but are above $59.2 \mathrm{~Hz}$ which is the first level to instantaneously shed load for UFLS setting in 
TABLE 3: Small-signal stability comparison for normal and N-3 contingency.

\begin{tabular}{lcccc}
\hline \multirow{2}{*}{ STN transfer (MW) } & \multicolumn{2}{c}{ System conditions } & & N-3 \\
& & & & \\
Normal & & Damped frequency (Hz) & Damping ratio (\%) \\
\hline 3800 & Damped frequency (Hz) & Damping ratio (\%) & 1.2043 & 3.75 \\
3900 & 1.2005 & 5.01 & 1.2053 & 3.60 \\
4000 & 1.2012 & 5.00 & 1.2063 & 3.40 \\
4100 & 1.2003 & 4.84 & 1.2072 & 3.20 \\
4200 & 1.2004 & 4.68 & 1.2071 & 3.06 \\
4300 & 1.1998 & 4.41 & 1.2076 & 2.97 \\
4400 & 1.1985 & 4.30 & 1.2082 & 2.92 \\
\hline
\end{tabular}

TPC system. This illustrates that the swing-down frequency does not reach the first level setting of UFLS. Therefore, electricity service is uninterrupted even in the most severe $\mathrm{N}-3$ contingency.

3.5. First-Stage SIPS Operation. The simulation identifies transient instability as the main factor in TPC system. We suggested TPC to install the SIPS against blackouts. TPC was completely responsible for the design and installation of the control system for the first-stage SIPS. The control system was installed in the control room of the Xinda power plant. After SIPS installation, functional testing is required to ensure each of its components is going to operate as designed under different system conditions. TPC takes the functional testing methods including functional element, integration, function, and system testing [19].

The operator in CDCC commands the plant operator to turn on generator rejection switches when the STN transfer exceeds $3700 \mathrm{MW}$ or encounters N-3 contingency. The fifteen turn-on/off switches on the control system panel enable 15 generators to be tripped as in Figure 7. From Table 2, if the STN transfer exceeds $4000 \mathrm{MW}$, then the system operator commands the Xinda plant operator to turn on ten switches connected to online operating generators. Accordingly, these ten generators are rejected in cases of N-3 contingencies.

\section{Second-Stage SIPS}

Two main reasons compelled TPC to construct a more reliable system-wide SIPS. One is the importance of delays in power construction programs and the other is all of the lessons learned from the large grid blackouts. The secondstage SIPS has the following features:

(1) full automation: real-time collection, calculation, decision making, and action;

(2) redundancy: the same double sets of equipment that each includes the independent control system and communication channel.

(3) global N-3 protection: all EHV N-3 contingencies are simulated and the critical ones are adopted to construct action tables;

(4) event-based action table: action tables elucidate countermeasures against transient instability. Establishing

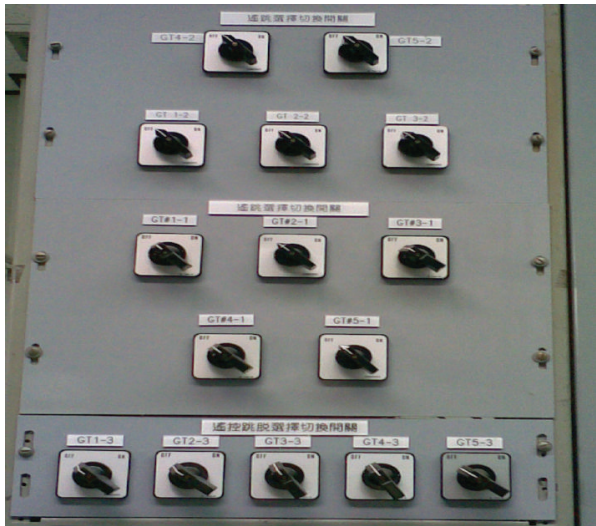

FIGURE 7: Generators tripping control panel of the first-stage SIPS.

the second-stage SIPS involves the following main tasks.

4.1. Architecture of Second-Stage SIPS. The architecture of the second-stage SIPS comprises five parts, as presented in Figure 8 . The detailed description is as follows.

(1) The first part is the operation control center which is the core of the SIPS. It is managed and controlled by CDCC operators and distributed both at Taipei and Kaohsiung CDCC.

(2) The second part is the EMS data collection server. It collects the interarea tie flow, substation load, and generator data from EMS via internal computer communication protocol (ICCP).

(3) The third part is the decision-making and action server which receives the relative information from EMS data collection server. It can identify contingencies to determine which action must be taken via front end processor (FEP) that can shed load, shed generator and energize bus-tie switch.

(4) The fourth part is the training and simulation server. It can change the settings of the action table in advance then to simulate the accuracy and availability. This part can also be the SIPS training site for operators. 


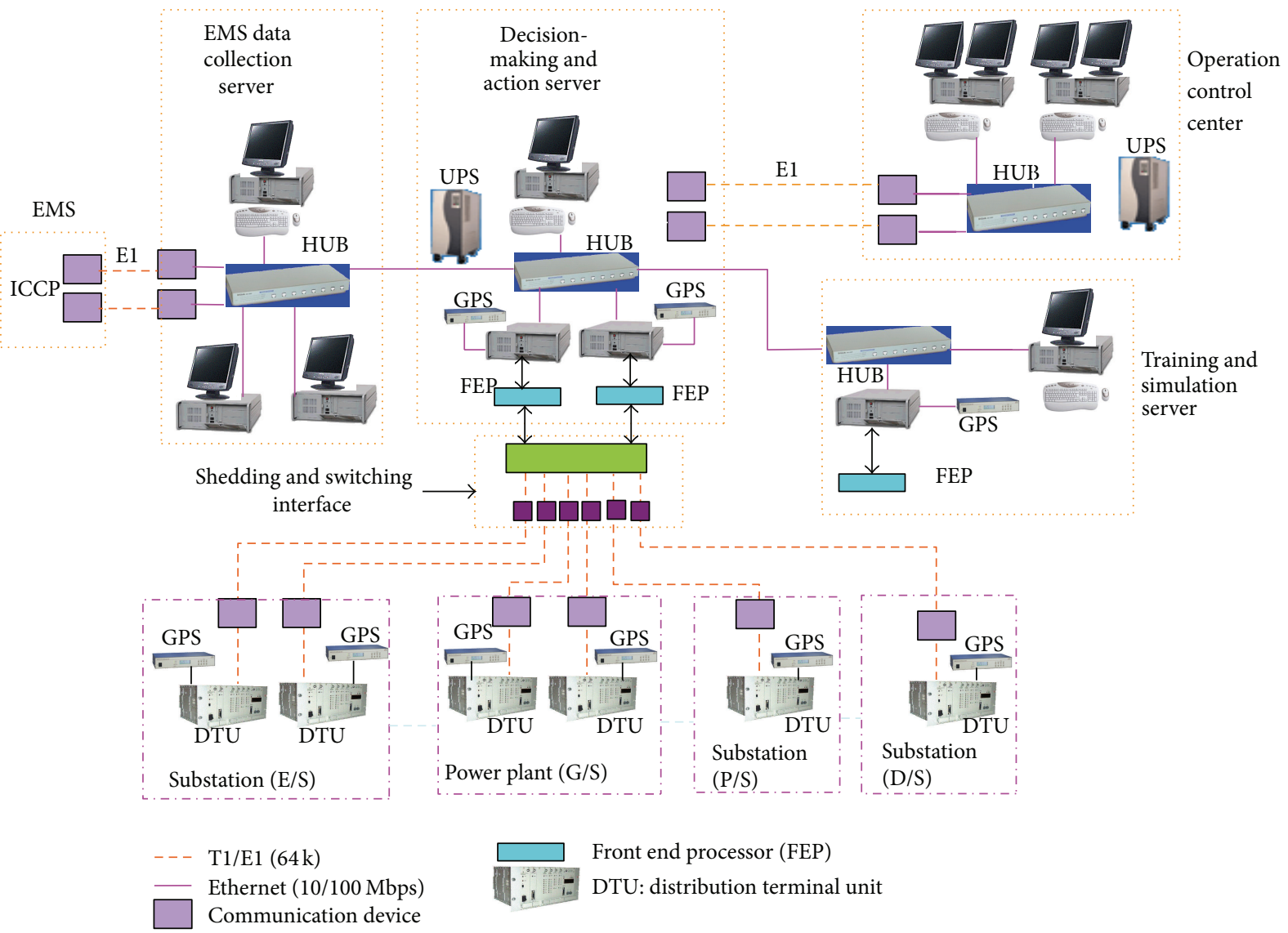

FIgURE 8: Architecture of the second-stage SIPS.

(5) The last part is the shedding and control interface. It contains three control modes which are load shedding, generator rejection, and bus-tie switching. The controlling objectives are 49 substations, three power plants, and two EHV bus-ties.

The first three parts have the redundant function which is equipped with the double independent supervisory consoles and communication channels. If one type of the double equipment fails or breaks down, the other takes over immediately. The communication media are E1/64 $\mathrm{k}$ protocol and Ethernet. The EMS data transfer is with internal computer communication protocol (ICCP). Time for devices is synchronized via GPS. The second-stage SIPS main equipment was located at Longtin EHV substation.

4.2. System Simulation. The design criteria are based on the $\mathrm{N}-3$ contingencies, which include N-2-1 and N-1-2. The " $\mathrm{X}$ " and "Y" for N- "X"- "Y" means the arming and triggering contingencies, respectively. Different load conditions, including peak, medium, and light, as well as power transfers, including south-to-central and central-to-north, are considered in system simulation. The kinds of system simulation contain the thermal violation, voltage violation, and small-signal and transient stability analyses. It takes much time to examine the system study results because of the magnitude and types.
The automatic processes for system study must be considered because hundreds of thousands cases must be simulated. The results of the system simulation are the look-up tables based on thermal violation, voltage violation, and smallsignal and transient stabilities. Table 4 presents the description of look-up table in peak load. On the top of the table, $\mathrm{C} 4$ and A37 are the table identifiers, which refer to arming and triggering contingencies. The look-up table comprises the following five main parts.

(1) Interarea transfer includes central-to-north (4600$4800 \mathrm{MW}$ ) and south-to-central (1800-3000 MW) power flow transfers.

(2) Load shedding includes level 1 shedding for transient instability and level 2 for thermal violation. Details of the number of shed substations and the shedding area are given. Most of the load shedding substations are in the north; some are in the center of Taiwan.

(3) Generator shedding is as follows. Three power plants may be rejected in cases of transient instability. Two plants are in the southern area and one in the central area.

(4) Bus-tie switch is switching action for bus-tie in the two EHV substations included; one is located in the northern and the other in the central area. 


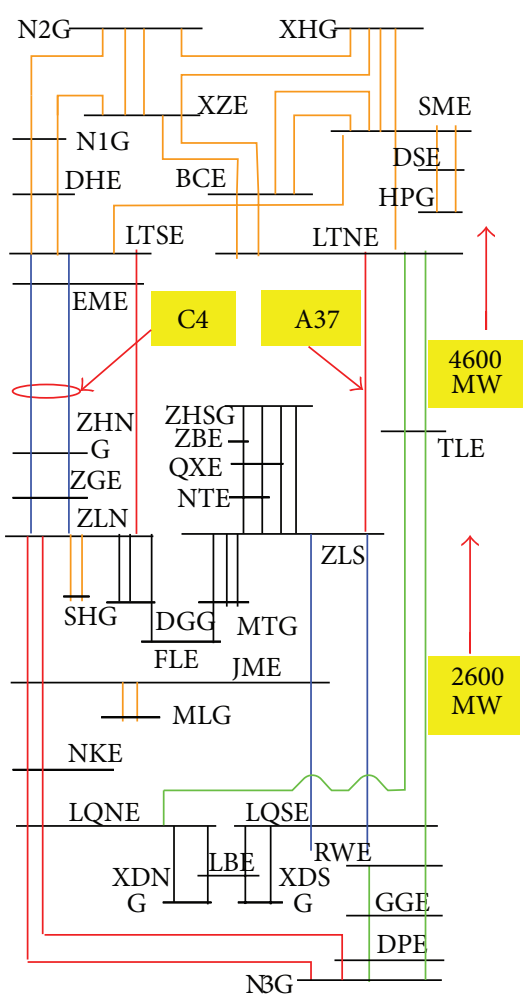

(a)

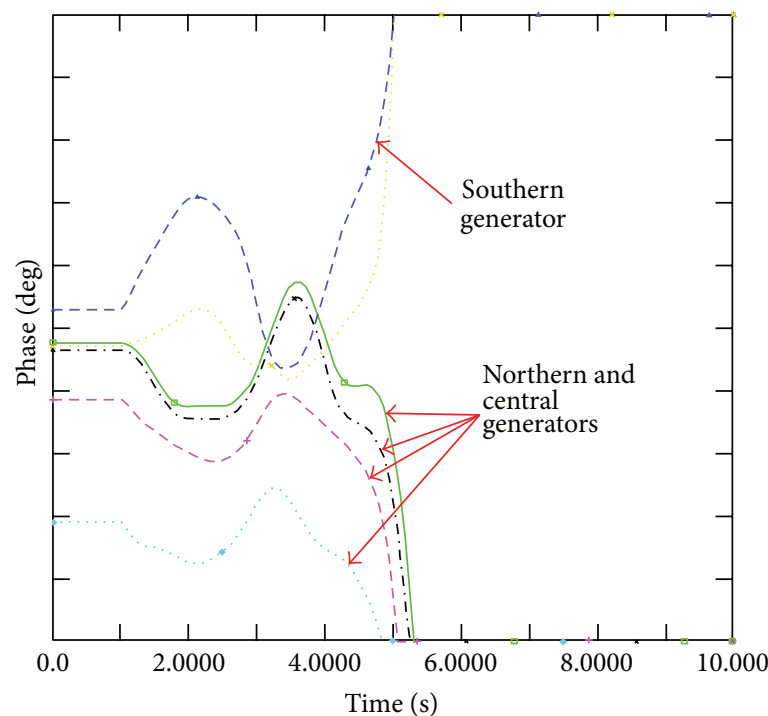

(b)

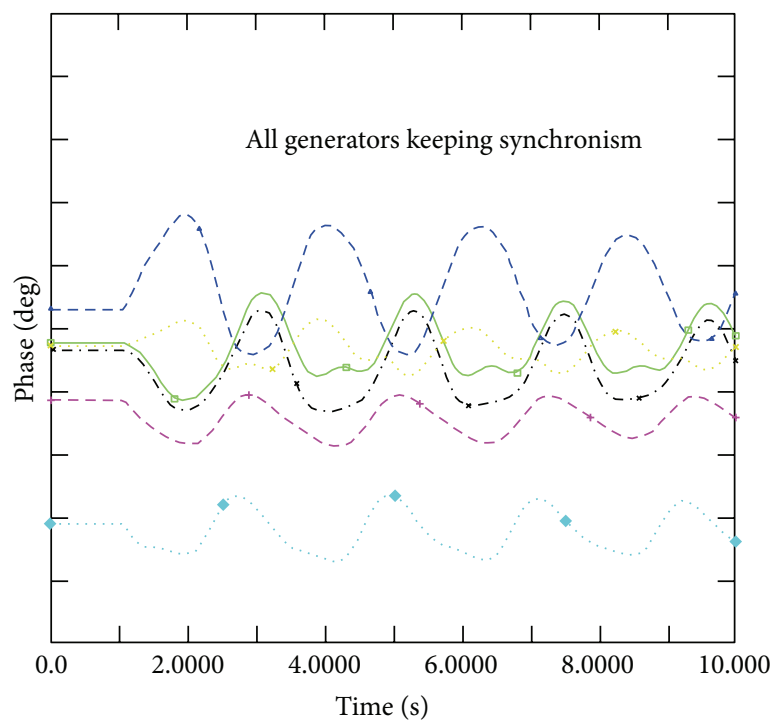

(c)

Figure 9: Taiwan $345 \mathrm{kV}$ transmission system and C4/A37 simulation results with/without SIPS action.

(5) Post-SIPS power flow check is as follows. The power flow is reexamined after an SIPS action in response to voltage and thermal violations.

We explain Table 4 with the interarea transfer $4600 \mathrm{MW}$ (central to north) and $2600 \mathrm{MW}$ (south to central) via C4/A37 contingencies as shown in Figure 9(a). When the interarea transfer is reaching $4600 \mathrm{MW}$ (central to north) and $2600 \mathrm{MW}$ (south to central) and C4 contingency (N2 contingency) happens, the SIPS enters arming condition, which means the power system is in stress. Then A37 contingency ( $\mathrm{N}-1$ contingency) happened which would cause power system to lose synchronism resulting from transient instability as shown in Figure 9(b). If SIPS takes actions by load shedding $(424.4+1043.65+402.142 \mathrm{MW})$ and generator shedding (Plant $1+$ Plant 2) after A37 contingency happened, 


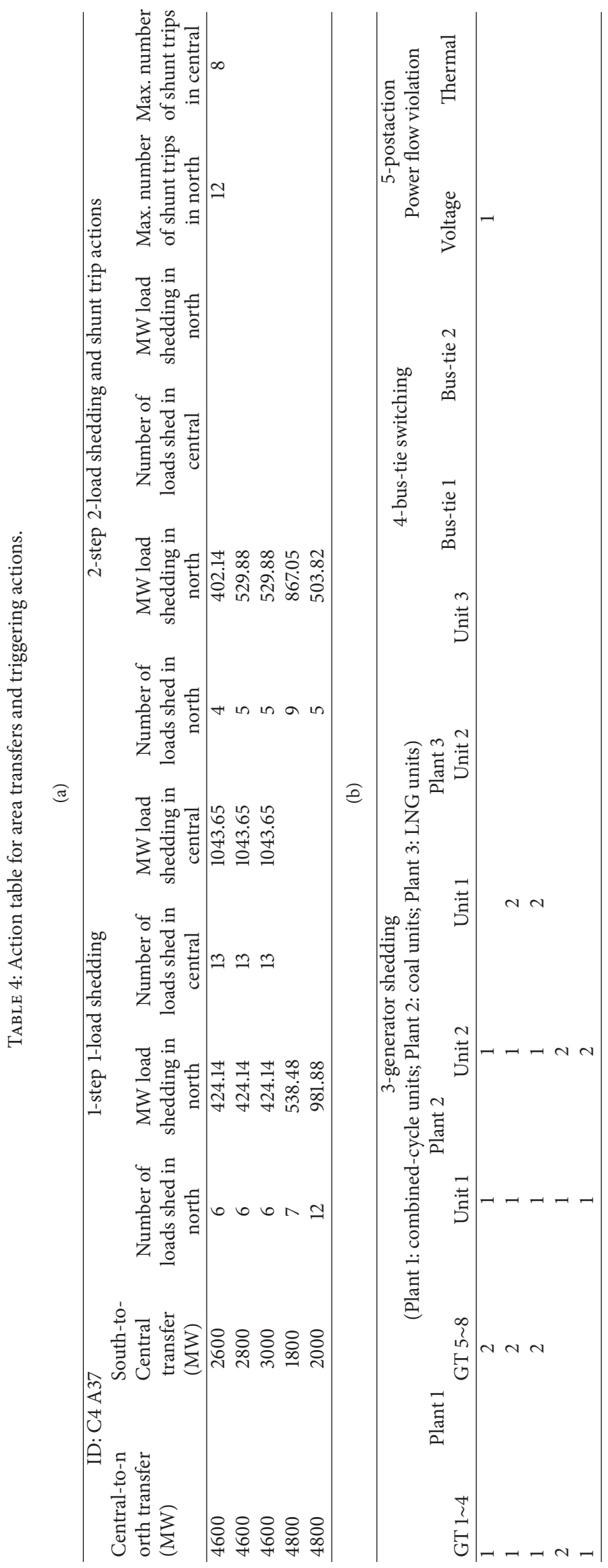




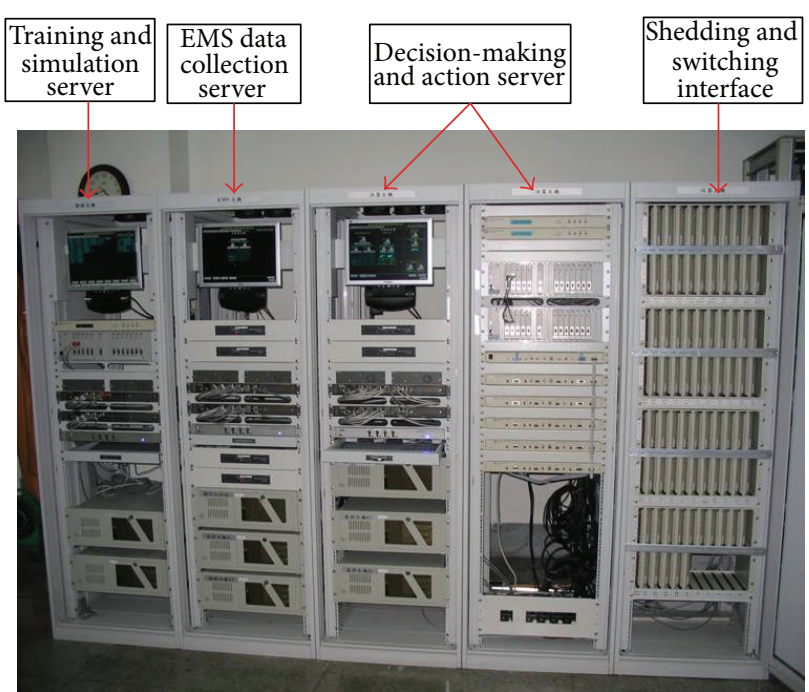

FIgURE 10: The control system main site of the second-stage SPS.

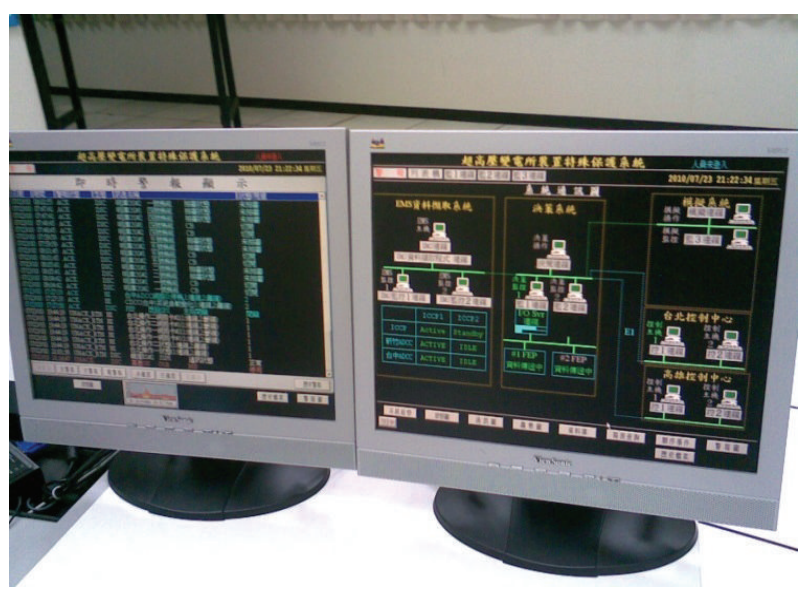

FIGURE 11: The second-stage SPS consoles sited at Taipei CDCC.

the power system would recover from the large disturbance as shown in Figure 9(c).

4.3. Operation and Maintenance. Functional and application testing are required to guarantee that each element could operate as designed under different system conditions [19]. The control system and communication channels must be examined and tested rigorously during testing period. 250millisecond action time is one of the important testing items. According to the testing results, the action times are less than 200 milliseconds. Figure 10 presents the control system main site of the second-stage SIPS located at Lungtan EHV substation. The components for each control server in Figure 10, from left to right, are the training and simulation server, the EMS data collection server, the decision-making and action server, and the shedding and switching interface. Figure 11 shows the consoles of the operation control center both located at Taipei/Kaohsiung control center. The SIPS consoles are very important for operators in dealing with extreme contingencies to maintain the grid security.

There was a real case for SIPS function that mitigated the stressed power system. On July 29, 2010, the central-tonorth and south-to-central transfers in TPC system reached $4850 \mathrm{MW}$ and $1900 \mathrm{MW}$. The SIPS alarm message that displayed the power system was in stress. From Table 4, the power system would be in arming condition if C4 contingency happened. As soon as the SIPS sent alarm message for stressed system, the CDCC operators had to take measures to mitigate the stressed condition. Therefore, The CDCC operators immediately started the $300 \mathrm{MW}$ gas turbine generators in the northern area. After 30 minutes, the central-to-north and south-to-central transfers decreased to $4550 \mathrm{MW}$ and $1600 \mathrm{MW}$. The grid recovered to normal from a stressed situation. The CDCC operators took the preventive measure to prevent the probable arming contingency, so that stopped the SIPS action in advance.

If the relative servers or communication channels break down, the self-healing function of SIPS starts to send cellular phone message to inform operators and maintenance engineer. As soon as the maintenance engineer receives the fixing message, he can process the problem with internet immediately. Therefore, the online sending fixing message is an important function for the second-stage SIPS.

\subsection{Performance. The main performances are the following.}

(1) Improvement of power system operation and reliability: the SIPS alarm message provides operators system conditions; that is, operators will make an adjustment to mitigate alert degree. It is easier to have blackout in case of extreme contingencies for this isolated and intensive power grid. After the installation of the SIPS, the power system conditions have been real-time monitored and controlled in advance. That would improve the power system operation and reliability.

(2) Create a prealert system for security: the operators will obtain the abnormal system information from the SIPS alarm message in advance. They will take proper measures to mitigate the stressed system to normal condition. This increases the power system security.

(3) Improvement of system simulation skills: in order to obtain the action table of the SIPS, hundreds of thousands power flow cases with contingencies must be simulated via program method. This improves the simulation skills of the power system.

(4) Fulfillment of the grid security goal for smart grid milestone in TPC: after the implementation of the SIPS, this helps TPC to achieve the goal in grid security and reliability group for its smart grid milestone.

\section{Conclusion}

This study describes the system simulation methods for SIPS implementation in an isolated, intensive, and islandtype power system to prevent blackout from extreme contingencies. The proposed two-stage SIPS with self-healing 
characteristics has been implemented and operating in the Taiwan power system. SIPS can remind operators to make an adjustment in advance to mitigate the urgent degree for the given arming condition. The limitation of first-stage SIPS is the specific protection only available for N-3 contingencies. The second-stage SIPS are designed to prevent all extreme contingencies and improve the first-stage SIPS. The look-up table includes system simulations of the load shedding, generator rejection, and bus-tie switching. The system simulations and implementation of the SIPS for preventing blackout in this independent power system are the main contribution. To create the function of prealert security system for operators is another contribution.

\section{Conflict of Interests}

The authors declare that there is no conflict of interests regarding the publication of this paper.

\section{Acknowledgment}

This work was supported in part by the National Science Council in Taiwan, under the project title: Caltech-Taiwan Collaboration on Energy Research-Uncertainty Mitigation for Renewable Energy Integration, Project no. NSC 101-3113P-008-001.

\section{References}

[1] J. A. Momoh, "Smart grid design for efficient and flexible power networks operation and control," in Proceedings of the IEEE/PES Power Systems Conference and Exposition (PSCE '09), March 2009.

[2] G. Andersson, P. Donalek, R. Farmer et al., "Causes of the 2003 major grid blackouts in North America Europe, and recommended means to improve system dynamic performance," IEEE Transactions on Power Systems, vol. 20, no. 4, pp. 1922-1928, 2005.

[3] Final Report on the disturbances of November 2006, https:// www.entsoe.eu/fileadmin/user_upload/_library/publications/ ce/otherreports/Final-Report-20070130.pdf.

[4] V. Madani, D. Novosel, S. Horowitz et al., "IEEE PSRC report on global industry experiences with System Integrity Protection Schemes (SIPS)," IEEE Transactions on Power Delivery, vol. 25, no. 4, pp. 2143-2155, 2010.

[5] C. F. Henville, "SIPS and stretched power systems," in Proceedings of the 10th IET International Conference on Developments in Power System Protection (DPSP '10), April 2010.

[6] IEEE Power System Relaying Committee, 2009, Global Industry Experiences with System Integrity Protection Schemes (SIPS), www.pes-psrc.org.

[7] V. Madani, D. Novosel, M. Begovic, and M. Adamiak, Application Considerations in System Integrity Protection Schemes (SIPS), GE Mag, 2008.

[8] M. Begovic, V. Madani, and D. Novosel, "System Integrity Protection Schemes (SIPS)," in Proceedings of the iREP SymposiumBulk Power System Dynamics and Control-VII, Revitalizing Operational Reliability, Charleston, South Carolina, August 2007.
[9] M. El-werfelli, H. A. Haddud, and R. Dunn, "Design of an optimized defence plan for a power system," in Proceedings of the 42nd International Conference on Large High Voltage Electric Systems (CIGRE '08), 2008.

[10] J. Hsu, K. Coceo, T. Isham, and B. Kokanos, "Use of special protection systems for major palo verde network hub congestion management in the U.S. Southwest," in Proceedings of the International Conference on Power System Technology (OWERCON '06), October 2006.

[11] J. Hsu, G. DeShazo, and W. Wong, "Use of special protection systems to overcome power congestion in the Western United States," in Proceedings of the International Conference on Power System Technology, pp. 1339-1343, 2002.

[12] A. Moshref, C. Henville, R. Curtis et al., "Design of a Special Protection System to Maintain System Security at High Import," in Proceedings of the IEEE Power Engineering Society General Meeting, pp. 311-319, July 2003.

[13] S. S. Ahmed, N. C. Sarker, A. B. Khairuddin, M. R. B. Abd Ghani, and H. Ahmad, "A scheme for controlled islanding to prevent subsequent blackout," IEEE Transactions on Power Systems, vol. 18, no. 1, pp. 136-143, 2003.

[14] Y.-J. Wang, C.-W. Liu, and Y.-H. Liu, "A PMU based special protection scheme: a case study of Taiwan power system," International Journal of Electrical Power and Energy Systems, vol. 27, no. 3, pp. 215-223, 2005.

[15] G. Trudel, "Hydro-quebec's defence plan against extreme contingencies," IEEE Transactions on Power Systems, vol. 14, no. 3, pp. 958-966, 1999.

[16] C.-H. Lee and S.-C. Hsieh, "Lessons learned from the power outages on 29 July and 21 September 1999 in Taiwan," IEE Proceedings: Generation, Transmission and Distribution, vol.149, no. 5, pp. 543-549, 2002.

[17] Taiwan Power Company, Taiwan Power Company 2012 Annual Report, 2012.

[18] Planning Criteria For Transmission System of Taiwan Power Company, TPC Regulation, 2011.

[19] A. Apostolov and B. Vandiver, "Requirements and tools for testing of system integrity protection schemes," in Proceedings of the 10th IET International Conference on Developments in Power System Protection (DPSP '10), April 2010. 


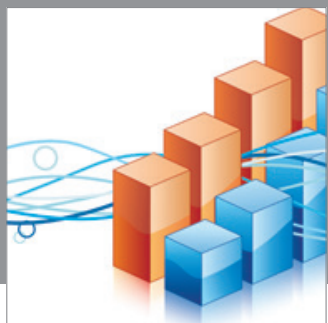

Advances in

Operations Research

mansans

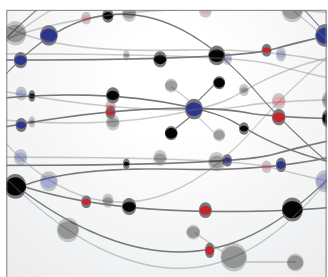

The Scientific World Journal
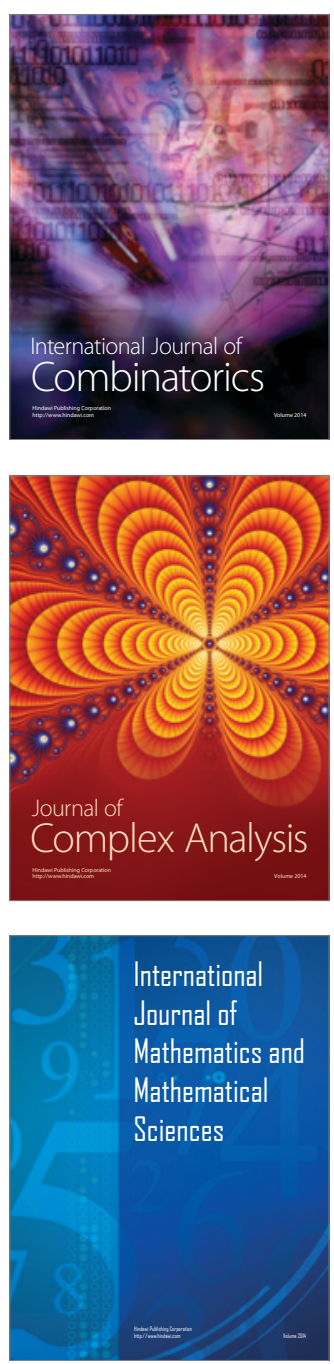
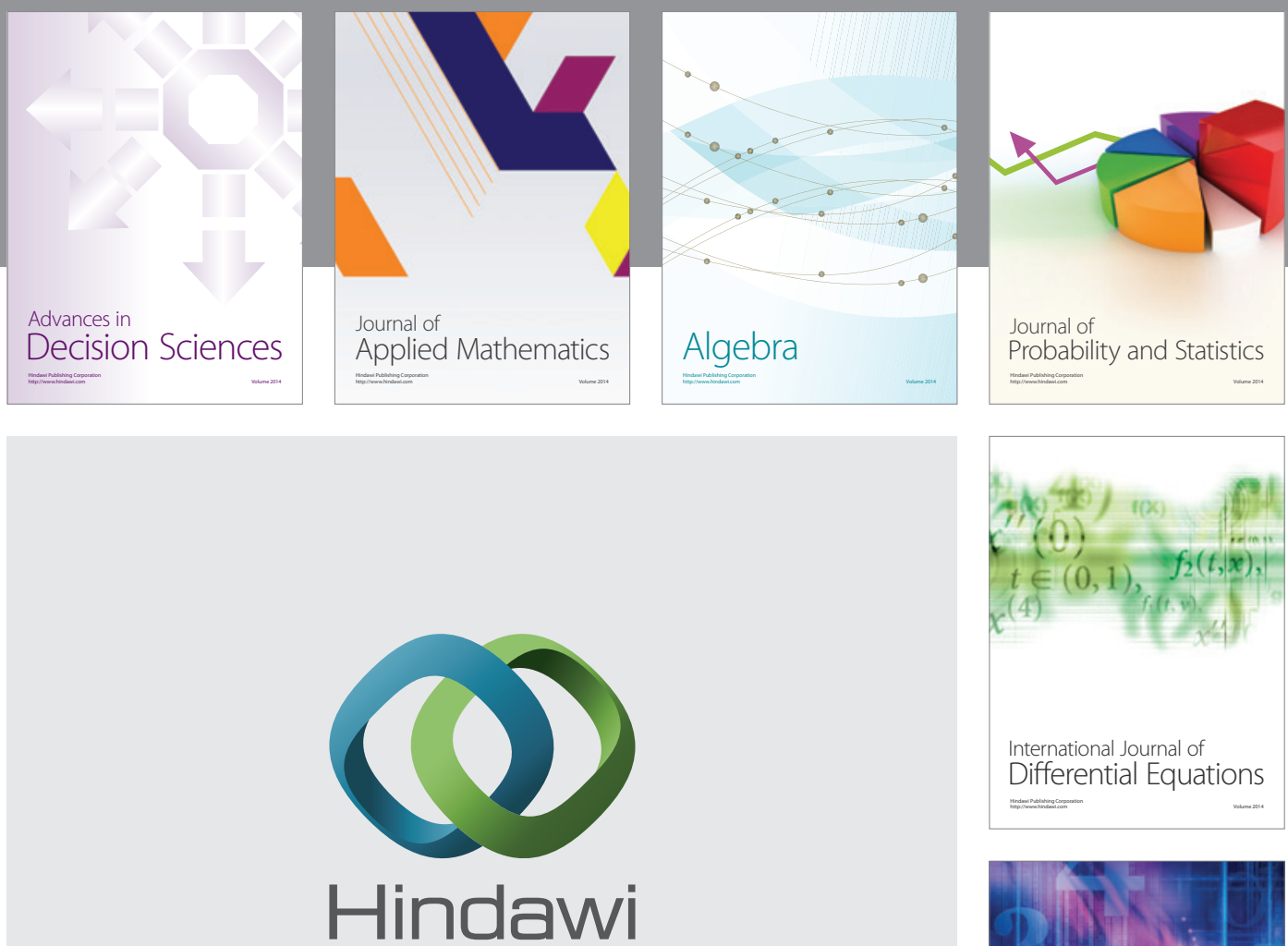

Submit your manuscripts at http://www.hindawi.com
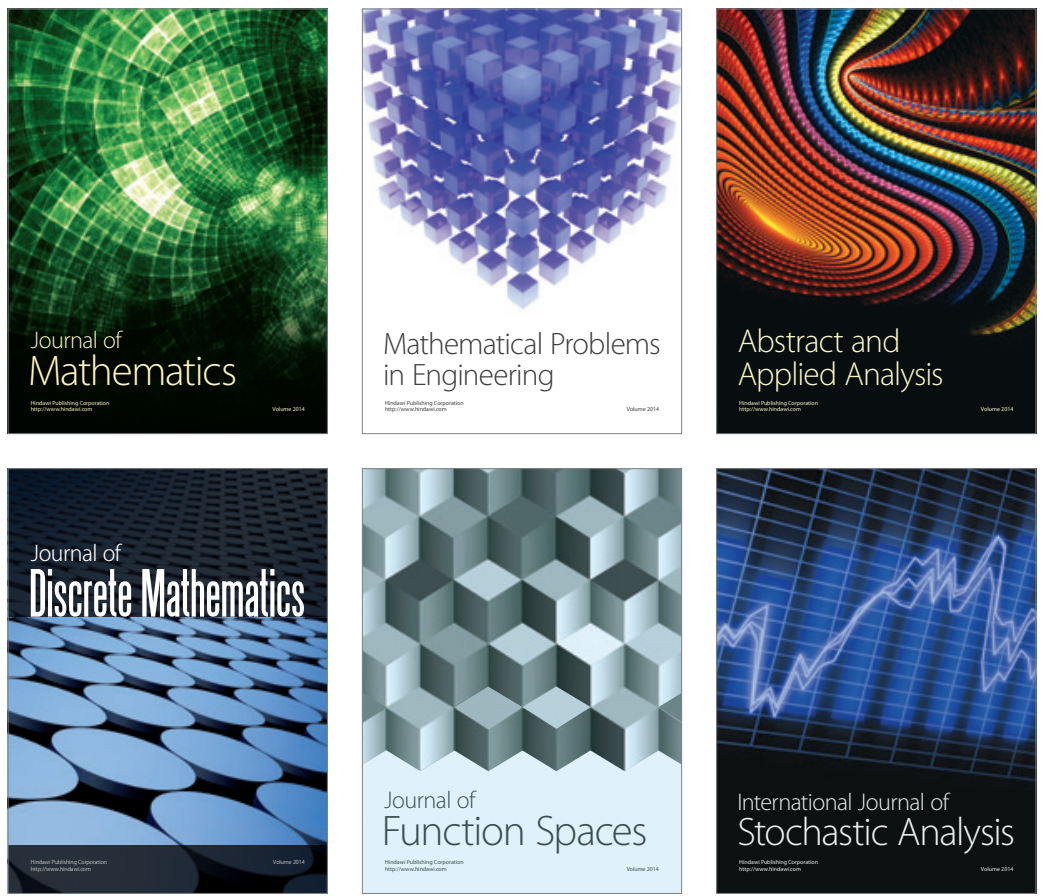

Journal of

Function Spaces

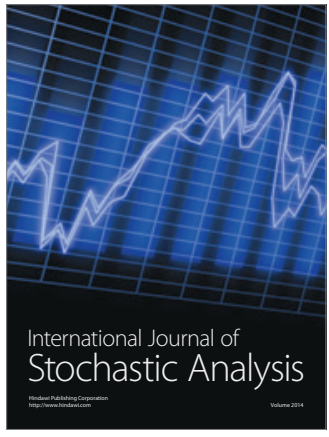

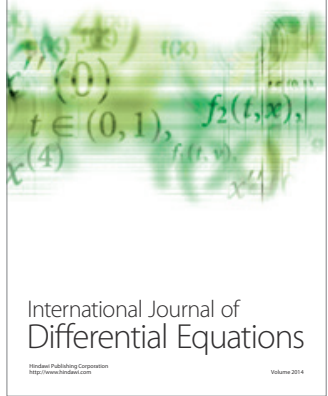
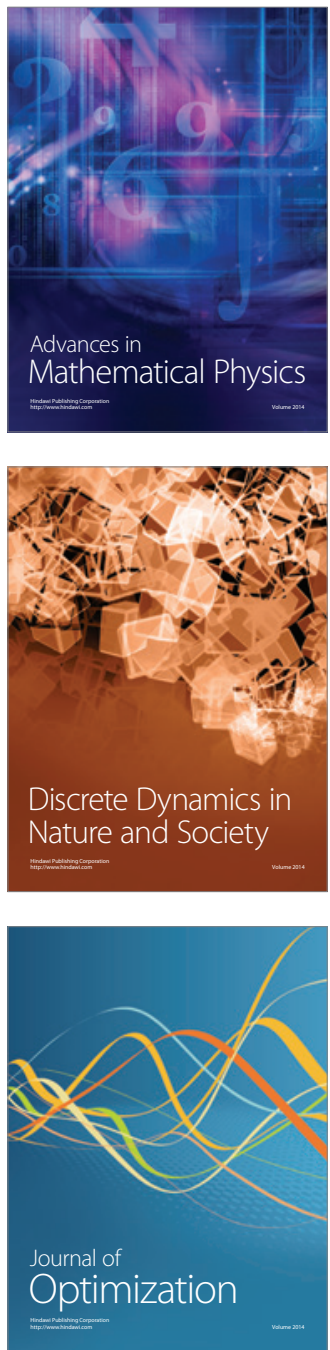